\title{
Envelhecimento: qualidade de vida e bem-estar das mulheres idosas
}

\begin{abstract}
Envelhecer é um processo natural em que ocorre redução de atividades laborais, psíquicas e biológicas. 0 envelhecimento envolve fatores diversos que fazem interligações multidisciplinares, caracterizando-se como um processo complexo. Objetivou-se, neste trabalho, analisar os fatores associados à qualidade de vida da mulher idosa, numa população geral, no ano de 2016. Trata-se de um estudo quantitativo, transversal e de caráter descritivo, realizada com 550 idosas, residentes no município de Guanambi (BA), no ano de 2016. Adotaram-se, como critérios de inclusão, idosas com idade maior ou igual a 60 anos, que estivessem conscientes e aceitaram participar da pesquisa, mediante assinatura do Termo de Consentimento Livre e Esclarecido. Utilizou-se como instrumento de coleta de dados formulário semiestruturado e o instrumento de avaliação da Qualidade de Vida da Organização mundial da Saúde (OMS), o World Health Organization Quality of Life (WHOQOL- bref) A análise dos dados obtidos foi realizada através do programa Stata versão 10. Vale ressaltar que foram cumpridas todas as exigências da resolução 466/12 do Ministério da Saúde. Das 550 idosas participantes da pesquisa, a maioria delas possuíam idade entre 60 a 70 anos (54,1\%), sem companheiro $(53,8 \%)$ e se declararam da raça/cor da pele negra. No tocante ao nível de escolaridade $(53,8 \%)$ das idosas cursou apenas o ensino fundamental e $39,5 \%$ eram analfabetas. Já para o quesito renda familiar $(60,4 \%)$ recebem um salário mínimo por mês. E para a cooperação familiar na renda da família $71,8 \%$ das idosas não tinha ajuda financeira, sendo sua renda a única para sustentar toda a família. As idosas moravam, em sua maioria, por mais de três familiares (32,4\%). $68,2 \%$ das idosas tem uma apercepção positiva quanto a sua qualidade de vida. Na análise estatística multivariada de regressão logística observou-se que as variáveis que se mantiveram no modelo final, como fatores associados à qualidade de vida das idosas, foram: a renda $(O R=1,62 ; \mathrm{IC}=1,16-2,26)$, a percepção da saúde $(O R=4,16 ; I C=2,77-6,25)$, lazer $(O R=2,03 ; I C=1,25-3,27)$ e a condição de moradia $(O R=3,10 ; I C=1,74-5,53)$. A qualidade de vida está diretamente associada a disponibilidade de recursos sociais utilizados e vivenciados no percurso da vida e no próprio processo do envelhecimento. Assim sendo, a carência ou excesso desses riscos socioeconômicos tem interferência e são indicadores de bem-estar na velhice, como por exemplo: a renda, o grau de escolaridade e condições de saneamento básico entre outros.
\end{abstract}

Palavras-chave: Fatores de risco; Envelhecimento populacional; Qualidade de vida.

\section{Aging: Quality of life and well-being of older women}

\begin{abstract}
Aging is a natural process in which there is a reduction in work, psychic and biological activities. Aging involves several factors that make multidisciplinary interconnections, characterized as a complex process. This study aimed to analyze the factors associated with the quality of life of elderly women in a general population in 2016. This is a quantitative, cross-sectional and descriptive study, conducted with 550 elderly residents of the municipality. de Guanambi (BA), in 2016. The inclusion criteria were elderly women older than or equal to 60 years old, who were aware and agreed to participate in the research, by signing the Informed Consent Form. A semi-structured form was used as a data collection instrument and the World Health Organization (WHO) Quality of Life assessment tool, the World Health Organization Quality of Life (WHOQOL- bref). Stata version 10. It is noteworthy that all the requirements of Resolution $466 / 12$ of the Ministry of Health were fulfilled. Of the 550 elderly participants in the research, most of them were aged between 60 and 70 years (54.1\%), without a partner (53.8\%) and declared themselves of the black race / skin color. Regarding the level of education (53.8\%) of the elderly only attended elementary school and $39.5 \%$ were illiterate. For family income $(60.4 \%)$ they receive a minimum wage per month. And for family cooperation in family income, $71.8 \%$ of the elderly women had no financial help, their income being the only one to support the whole family. Most elderly women lived for more than three family members (32.4\%). $68.2 \%$ of the elderly women have a positive perception regarding their quality of life. In the multivariate statistical analysis of logistic regression, it was observed that the variables that remained in the final model, as factors associated with the quality of life of the elderly, were: income $(\mathrm{OR}=1.62 ; \mathrm{Cl}=1.16-2.26)$. $)$, health perception $(\mathrm{OR}=4.16$ $\mathrm{Cl}=2.77-6.25)$, leisure $(\mathrm{OR}=2.03 ; \mathrm{Cl}=1.25-3.27)$ and housing condition $(\mathrm{OR}=3.10 ; \mathrm{Cl}=1.74-5.53)$. Quality of life is directly associated with the availability of social resources used and experienced in the course of life and in the aging process itself. Thus, the lack or excess of these socioeconomic risks has interference and are indicators of well-being in old age, such as income, education and basic sanitation conditions, among others.
\end{abstract}

Keywords: Risk factors; Population-ageing; Quality of life.

Topic: Enfermagem em Saúde Pública

Reviewed anonymously in the process of blind peer.

Wellington Pereira Rodrigues (iD)

Centro Universitário AGES, Brasil

http://lattes.cnpq.br/2798105983752891

http://orcid.org/0000-0002-0993-6871

wellington life@live.com

Priscila Dantas Gonçalves (iD

Centro Universitário AGES, Brasil

http://lattes.cnpq.br/7078066094809493

http://orcid.org/0000-0002-1849-0226

prisciladantasg@hotmail.com
Received: 10/11/2018

Approved: 11/01/2019

\section{Referencing this:}

RODRIGUES, W. P.; GONÇALVES, P. D.. Envelhecimento: qualidade de vida e bem-estar das mulheres idosas. Scire Salutis, v.9, n.1, p.30-36, 2019. DOI: http://doi.org/10.6008/CBPC2236-9600.2019.001.0004 


\section{INTRODUÇÃO}

Envelhecer é um processo natural em que ocorre redução de atividades laborais, psíquicas e biológicas. Com o avanço da idade, a pessoa idosa tende a ficar gradativamente vulnerável, e em alguns casos, dependente de terceiros para exercer as suas atividades vitais. Por critério de definição, o Estatuto do Idoso considera idosa aquela que tem idade igual ou superior 60 anos, e enfatiza o envelhecimento como um direito personalíssimo, e sua proteção como um direito social (AMARAL, 2015).

O envelhecimento envolve fatores diversos que fazem interligações multidisciplinares, se caracterizando como um processo complexo. O aumento do número de idosos tem ocorrido de maneira global, decorrente de alterações demográficas nas últimas décadas, estando constantemente associado, às transformações do perfil epidemiológico e das características econômicas e sociais das populações (ALBERTE, 2015).

A população idosa do Brasil vem aumentando consideravelmente, segundo a Política Nacional do Idoso e o Estatuto do Idoso, o percentual de pessoas idosas, é aproximadamente 10,8\% da população total, ou seja, 20.590.599 milhões. Em 2010, desse universo, o gênero feminino representava 55,5\% comparado com o gênero masculino com 44,5\%. Esse acréscimo reflete em maior quantitativo do envelhecimento da mulher, e a razão para esse fato é o processo decorrente da expectativa de vida, maior nas mulheres, que vivem em média de 8 anos a mais que os homens (CAMPOS, 2015). Nesse sentido, é necessário que o idoso se mantenha ativo e envolvido com as atividades diárias, através do compartilhamento de atividades físicas, sociais e intelectuais, conservando sua autonomia e independência (CABRAL, 2013).

O envelhecimento ativo é "o processo de otimização das oportunidades de saúde, participação e segurança, com o objetivo de melhorar a qualidade de vida à medida que as pessoas ficam mais velhas". Desse modo, o sentido de envelhecer é oportunizar ao indivíduo, a escolha por hábitos de vida saudáveis, proporcionando o controle da saúde e condicionando uma melhora na qualidade de vida, decorrente do bem-estar emocional, que envolve o relacionamento social (CARTAXO et al., 2012).

A qualidade de vida é um reflexo dos fatores subjetivos, como o amor, inserção e realização social, liberdade e dos fatores objetivos, como alimentação lazer, educação, saúde e habitação, que proporciona bem-estar conforto, realização individual e coletiva (CAMPOS, 2015). A qualidade de vida pode ser definida como a "percepção do indivíduo de sua posição na vida em relação ao contexto e sistemas de valores nos quais se insere bem como seus objetivos, expectativas, padrões e preocupações" (CAMPOS, 2015). Nota-se que esse é um conceito complexo, compreendendo a saúde física, vínculo sociais, estado psíquico, nível de independência e a interação do indivíduo com o meio ambiente que o cerca.

Diante disso, o presente estudo objetivou analisar os fatores associados à qualidade de vida da mulher idosa, numa população geral, no ano de 2018. A qualidade de vida está diretamente associada à disponibilidade de recursos sociais utilizados e vivenciados no percurso da vida e no próprio processo do envelhecimento. Assim sendo, a carência ou excesso desses riscos socioeconômicos tem interferência e são indicadores de bem-estar na velhice, como por exemplo: a renda, o grau de escolaridade e condições de 
saneamento básico entre outros. Assim, destaca-se a relevância científica e social de se investigar as condições que interferem no bem-estar na senescência, e os fatores associados à qualidade de vida de idosos, sobretudo da mulher idosa, no intuito de criar alternativas de intervenção e propor ações e políticas na área da saúde, buscando atender às demandas da população que envelhece (CAUDURO, 2013).

\section{MATERIAL E MÉTODOS}

Tratou-se de um estudo quantitativo, transversal e de caráter descritivo. Nesse contexto, a pesquisa qualitativa salienta que tudo pode ser medido, ou seja mensurável em números, opiniões e informações para agrupá-los e analisá-Ios (PRODANOV, 2013). O estudo quantitativo é "o ato de medir e analisar as relações casuais entre as variáveis" (DENZIN, 2006), de modo que há a permissa de análise de causa e efeito.

A pesquisa foi realizada no município de Guanambi, estado da Bahia, situado a $796 \mathrm{~km}$ de sua capital, Salvador possuindo uma área territorial de $1.301,80 \mathrm{~km}^{2}$. O último censo demográfico realizado em 2010 para este município constatou 78.833 habitantes, dos quais o idoso representa 8.618 ou seja cerca de $10,93 \%$ da população, desse universo, as idosas são 4.645 representando 5,89\% da população total.

A coleta de dados ocorreu de dezembro de 2017 a março de 2018. A amostra probabilística simples em que todos os elementos da amostra têm a chance de escolha, e com base no número total das idosas, a amostra final foi de 550 idosas. Assim, o sorteio ocorreu por bairro. Para critérios de inclusão do estudo foram selecionadas idosas com idade maior ou igual a 60 anos, que estivessem conscientes e aceitaram participar da pesquisa, mediante assinatura do Termo de Consentimento Livre e Esclarecido. Para efetuar a coleta de dados, a idosa foi abordagem em suas residências, após mapeamento obtido mediante cadastro nas Unidades de Saúde da Família dos respectivos bairros.

A coleta de dados foi realizada em quatorze bairros do município. $O$ instrumento utilizado na pesquisa, foi um formulário semiestruturado, que buscou contemplar as informações dos aspectos de saúde e sociodemográfico das idosas, e o instrumento de avaliação da Qualidade de Vida da Organização mundial da Saúde (OMS), o World Health Organization Quality of Life (WHOQOL- bref) resumida na versão em português. Quanto aos instrumentos supracitados foi utilizada a versão em português resumida o WHOQOLbref que é constituído por 26 perguntas pertencentes a quatros domínios são eles: físico, psicológico, relações sociais e meio ambiente (FEHLBERG, 2013).

Para análise da percepção da qualidade de vida foi utilizado os escores adequado e inadequado. Foi feita uma análise estatística univariada, com cálculos de $\chi 2$, para avaliar as variáveis sociodemográficas citadas anteriormente e sua relação com a percepção da qualidade de vida. Realizou-se também uma análise de regressão logística para identificar os principais fatores associados a qualidade de vida das idosas, na qual a variável dependente foi a percepção da qualidade de vida e as variáveis independentes foram as que se mostraram significativas na análise univariada, assim como as variáveis que não foram significativas. Os dados foram tabulados utilizando o programa de computador Microsoft Excel ${ }^{\circledR}$, analisado por meio do programa estatístico Stata versão 10. 
Todas as fases deste projeto de pesquisa foram realizadas em consonância com as questões éticolegais da resolução n. 466/12 do Conselho Nacional de Saúde que regulamenta as pesquisas envolvendo seres humanos no Brasil. O presente projeto foi aprovado pelo Comitê de Ética e Pesquisa da Faculdade Independente do Nordeste (FAINOR), sob o protocolo CAAE: 50695415.5.0000.5578.

\section{RESULTADOS}

Das 550 idosas participantes da pesquisa a maioria delas possuíam idade entre 60 a 70 anos $(54,1)$, sem companheiro $(53,8 \%)$ e se declararam da raça/cor da pele negra, que é junção de pardas e pretas (54,4\%). Quanto ao nível de escolaridade, a maioria $(53,8 \%)$ das idosas cursou apenas o ensino fundamental e 39,5\% eram analfabetas. No que se refere à renda familiar $60,4 \%$ recebem um salário mínimo por mês. Quanto a cooperação familiar na renda da família $71,8 \%$ das idosas não tinha ajuda financeira, sendo sua renda a única para sustentar toda a família. As idosas moravam, em sua maioria, por mais de três familiares (32,4\%). Quanto ao tipo de moradia, 95,1\% das idosas possuem casa própria, com mais de seis cômodos $(70,7 \%)$, sendo que $75,6 \%$ referem ter dois banheiros em sua residência.

Os resultados mostraram que $68,2 \%$ das idosas tem uma apercepção positiva quanto a sua qualidade de vida. Na análise estatística univariada apontou apenas a renda $(p: 0,002)$ como variável estatisticamente associada à percepção da qualidade de vida. Observou-se que $69,8 \%$ dos sujeitos com renda familiar com mais de três salários mínimos apresentaram percepção de qualidade de vida negativo.

$\mathrm{Na}$ análise das condições de vida, cinco variáveis apresentaram significância estatística relacionada com a percepção da qualidade de vida: percepção da saúde $(p: 0,000)$, recursos financeiros $(p: 0,000)$, lazer ( $p: 0,000)$, condições de moradia $(p: 0,000)$ e transporte $(p: 0,012)$. Essas variáveis identificadas como significativas na análise, foram utilizadas no modelo multivariado de regressão logística, juntamente com as variáveis que não foram significativas, coletadas no questionário sociodemográficas.

$\mathrm{Na}$ análise estatística multivariada de regressão logística pode-se observar que as variáveis que se mantiveram no modelo final, como fatores associados à qualidade de vida das idosas, foram: a renda $(\mathrm{OR}=$ 1,62; IC =1,16-2,26), a percepção da saúde ( $O R=4,16 ; I C=2,77-6,25)$, $\operatorname{lazer}(O R=2,03 ; I C=1,25-3,27)$ e a condição de moradia $(O R=3,10 ; I C=1,74-5,53)$. As demais variáveis não se mantiveram no modelo multivariado.

\section{DISCUSSÃO}

O envelhecimento saudável depende da relação multidimensional de diversos fatores relacionados às idosas, tais como renda, escolaridade, sexo, estado civil e lazer, que podem influenciar de forma direta na sua qualidade de vida (CAUDURO, 2013). Estudos constatam que, a qualidade de vida está associada ao contexto socioeconômico das pessoas. As condições socioeconômicas desfavoráveis para as idosas, sejam por necessidade de auxílio financeiro, físico ou afetivo, afetam diretamente a forma de cada idosa vivenciar com seus familiares, pois criam-se grandes laços de dependência, o que compromete o bem-estar das mesmas (CHAIMOWICZ, 2013). 
Por outro lado, melhores condições socioeconômicas, pode influenciar positivamente no estado psicológico, nos domínios físicos e nas relações sociais, colaborando para que estas, residam sozinhas (KÜCHEMANN, 2012). Essa dependência pode ser resultante do ambiente físico em que o idoso está inserido, do grau de proporção de segurança dos locais, que são suscetíveis à depressão e ao isolamento social, sendo que a última, tem conexão com o enfraquecimento da saúde física e mental (KHOURY, 2014).

Em um estudo populacional realizado em São Paulo, evidenciou-se a assiduidade de sintomas de depressão relacionados a menores rentabilidade, menores níveis de alfabetização e a inferioridade na autopercepção do estado de saúde. Outro estudo feito em Jequié-Bahia, mostrou que $70 \%$ das idosas apresentavam baixo nível socioeconômico, se enquadrando nas classes econômicas ' $D$ ' e ' $E$ '. Sendo que 60,6\% dessas idosas eram aposentadas, e apontavam a aposentadoria como a principal fonte de sobrevivência, além do mais, 13,9\% estavam inseridas no mercado de trabalho, como diarista, para suprir as necessidades básicas (MACHADO, 2015).

Nessa faixa etária, a fonte de renda é predominantemente a aposentadoria, de no máximo 2,5 salários mínimos, fazendo com que muitas idosas vivam em pobreza extrema, enquanto outras, para garantir suas condições de sobrevivência e não reduzir seu padrão de vida, são obrigados a permanecer no trabalho (FREITAS et al., 2012).

Entende-se que esta condição econômica, está relacionada ao menor acesso ao serviço de saúde e vulnerabilidade social, aos fatores que ocasiona o comprometimento funcional e a maior incidência de fatores de risco e suscetibilidade a problemas de saúde (VISENTIN et al., 2015). Nessa percepção, a baixa renda significa perda da eficiência, para cuidar de si próprio e na convivência social, uma vez que a falta de recursos para o autocuidado e ao pensamento de desigualdade social aumentando consideravelmente a proporção de doenças crônicas não transmissíveis, como a dependência ou depressão (OLIVEIRA, 2013).

Estudos demonstram que, as idosas que possuem uma renda familiar com menos de dois salários, tem maiores chances de residirem sozinhas do que aquelas com renda maior que dois salários mínimos. Porém, em relação a renda individual, quanto maior for a renda, maior será a probabilidade de a idosa viver sozinha (VIEIRA et al., 2012). Nos países desenvolvidos, o número de idosas que vivem com os filhos diminui consideravelmente com o passar da idade. De outro modo, os países em desenvolvimento, mesmo com a longevidade, continuam elevada a proporção de idosas que moram com os filhos (MODENEZE et al., 2013).

A maioria dos estudos mostram a predominância da feminilização na velhice, observada na pirâmide etária populacional, tanto de países em desenvolvimento, quanto em desenvolvidos, resultante do maior uso de bebidas alcoólicas e tabaco, menor assistência médica e exposição dos jovens homens à mortalidade decorrente de causas externas como trânsito e homicídio. Essa prevalência do gênero feminino é um fenômeno internacional, a exemplo de estudos em Alemanha, Canadá e, especialmente com o passar da idade (DAWALIBI, 2014).

Uma pesquisa realizada na Grã-Bretanha, relata que a mulher é mais propensa a viver sozinha do que o homem. Sendo que $30 \%$ das mulheres entre a faixa etária de 65 e 74 anos, vivem sozinhas, na medida que 
20\% dos homens nessa mesma idade. Esse fenômeno ocorre normalmente porque os homens nessa mesma faixa etária e condição da mulher, preferem relacionar com um cônjuge mais jovem (SANTOS, 2013).

Em estudo no Irã, para análise de fatores relacionados à qualidade de vida, verificou-se que a infelicidade e a dificuldade de relacionamento social entre idosas era decorrente do menor nível educacional evidenciando uma maior prevalência de analfabetismo entre mulheres idosas, sendo aproximadamente 37, 4\% entre mulheres e 32, 2\% entre homens, no Brasil em 2000 (SILVA, 2013).

Nota-se que, a mulher, principalmente na terceira idade, vem sendo excluída da participação política e social. Nesse contexto, o casamento permite a mulher a segurança econômica inerente as exigências sociais. Porém, observa-se em estudos o aumento progressivo de idosas sem cônjuge, a exemplo das solteiras, separada e viúvas (VITORINO, 2013).

Nesse sentido, a morte do companheiro de longos anos, significa uma tragédia que resulta comumente em um luto prolongado, ocasionando sentimento de tristeza, solidão e adoecimento. Em contrapartida, os idosos casados possuem um nível maior de expectativa de vida e de bem-estar do que osnão casados, devido a assistência e os cuidados que o cônjuge presta em situações de doença e nos enfraquecimentos físicos (SILVA, 2014).

Outros fatores que são essenciais ao envelhecimento com independência e autonomia, são as atividades físicas e de lazer, promovendo a socialização da idosa, tornando-a ativa, auxiliando na percepção de controle e diminuição de sintomas depressivos, proporcionando um desenvolvimento da saúde mental e física (SOUZA, 2015). A inexistência ou limitação do lazer é um fator de agravo e que ocasiona o sedentarismo, comprometendo assim, a capacidade funcional da idosa (VAGETTI et al., 2013).

\section{CONCLUSÕES}

Portanto, é importante considerar que, o envelhecimento da população traz a necessidade de reorientação das políticas públicas, tanto nas questões sociais quanto previdenciárias, e, sobretudo de saúde, pois o processo de envelhecimento vem acompanhado de alterações físicas, sociais e mentais. Tendo em vista que a qualidade de vida está diretamente associada a disponibilidade de recursos sociais utilizados e vivenciados no percurso da vida e no próprio processo do envelhecimento. Assim sendo, a carência ou excesso desses riscos socioeconômicos tem interferência e são indicadores de bem-estar na velhice, como por exemplo: a renda, o grau de escolaridade e condições de saneamento básico entre outros.

\section{REFERÊNCIAS}

ALBERTE, J. S.; RUSCALLEDA, R. M.; GUARIENTO, M.. Qualidade de vida e variáveis associadas ao envelhecimento patológico. Revista da Sociedade Brasileira de Clínica Médica, v.13, n.1, p.32-39, 2015.

AMARAL, T. M. R.; MELO, E. M.; OLIVEIRA, G. L.. Comparação do perfil de idosos ativos e não ativos do Programa Bolsa Família. Revista Brasileira de Geriatria e Gerontologia, Rio de Janeiro, v.18, n.2, p.351-360, 2015.
CABRAL, R. W. L.; SANTOS, S. R.; MENEZES, K. D. N. B.; ALBUQUERQUE, A. V.; MEDEIROS, A. L.. Fatores Sociais E Melhoria Da Qualidade De Vida Dos Idosos: Revisão Sistemática. Revista de Enfermagem da UFPE, Recife, v.7, n.5, p.1434-1442, 2013.

CAMPOS, A. C. V.; FERREIRA, E. F.; VARGAS, A. M. D. Determinantes do envelhecimento ativo segundo a qualidade de vida e gênero. Ciência \& Saúde Coletiva, v.20, n.7, p.2221-2237, 2015. 
CARTAXO, H. G. O.; SILVA, E. A. P. C.; SANTOS, A. R. M.; SIQUEIRA, P. G. B. S.; PAZZOLA, C. M.; FREITAS, C. M. S. M.. Percepção de idosas sobre o envelhecimento com qualidade de vida: subsídio para intervenções públicas. Rene, v.13, n.1, p.158-168, 2012.

CAUDURO, A.; GONÇALVES, A. J.; CAUDURO, M. H. F.. Fatores Associados A Morar Sozinho E Suas Diferenças Regionais Em Idosos Residentes De Porto Alegre E Manaus. Estudos Interdisciplinares sobre Envelhecimento, Porto Alegre, v.18, n.2, p.349-365, 2013.

CHAIMOWICZ, F.. Saúde do Idoso. 2 ed. Belo Horizonte: Nescon, 2013.

DAWALIBI, N. W.; GOULART, M. M.; PREARO, L. C.. Fatores relacionados à qualidade de vida de idosos em programas para a terceira idade. Ciência \& Saúde Coletiva, v.19, n.8, p.3505-3512, 2014

DENZIN, N. K.; LINCOLN, Y. S.. Introdução: a disciplina e a prática da pesquisa qualitativa. In: DENZIN, N. K.; LINCOLN, Y. S.. O planejamento da pesquisa qualitativa: teorias e abordagens. 2 ed. Porto Alegre: Artmed, 2006.

FEHLBERG, M. P. A.. Mulheres idosas e suas histórias de vidas sofridas: direito a adoecer com cidadania; quem sabe um dia. Kairós Gerontologia, v.16, n.5, p.269-292, 2013.

FREITAS, C. M. S. M.; MOURA, P. V.; SILVA, E. A. P. C.; CARTAXO, H. G. O.; SILVA, P. P. C.; CAMINHA, I. O.; SMETHURST, W. S.. Identidade do idoso: representações no discurso do corpo que envelhece. Estudos Interdisciplinares sobre o Envelhecimento, Porto Alegre, v.17, n.1, p.19-35, 2012.

KHOURY, H. T. T.; SÁ-NEVES, Â. C.. Percepção de controle e qualidade de vida: comparação entre idosos institucionalizados e não institucionalizados. Revista Brasileira de Geriatria e Gerontologia, Rio de Janeiro, v.17, n.3, p.553-565, 2014.

KÜCHEMANN, B. A.. Envelhecimento populacional, cuidado e cidadania: velhos dilemas e novos desafios. Sociedade e Estado, v.27, n.1, p.165-179, 2012.

MACHADO, A.; VIEIRA, M. C. U.. Impacto de fatores socioeconômicos na funcionalidade da pessoa idosa portadora de condições crônicas. Revista de Enfermagem da UFSM, v.5, n.1, p.81-91, 2015.

MODENEZE, D. M.; MACIEL, E. S.; JÚNIOR, G. B. V.; SONATI, J. G.; VILARTA, R.. Perfil epidemiológico e socioeconômico de idosos ativos: qualidade de vida associada com renda, escolaridade e morbidades. Estudos Interdisciplinares sobre o Envelhecimento, Porto Alegre, v.18, n.2, p.387-399, 2013.

OLIVEIRA, M. P. F.; NOVAES, M. R. C. G.. Perfil socioeconômico, epidemiológico e farmacoterapêutico de idosos institucionalizados de Brasília, Brasil. Ciência \& Saúde Coletiva, v.18, n4, p.1069-1078, 2013.

PRODANOV, C. C.; FREITAS, E. C.. Metodologia do trabalho científico: métodos e técnicas da pesquisa e do trabalho acadêmico. 2 ed. Novo Hamburgo: Feevale, 2013.

SANTOS, G. S.; CUNHA, I. C. K. O.. Avaliação da qualidade de vida de mulheres idosas na comunidade. Revista de Enfermagem do Centro Oeste Mineiro, v.4, n.2, p.11351145, 2014.

SILVA, M. E.; CRISTIANISMO, R. S.; DUTRA, L. R.. Perfil epidemiológico, sociodemográfico e clínico de idosos institucionalizados. Revista de Enfermagem do Centro Oeste Mineiro, v.3, n.1, p.569-576, 2013.

SILVA, P. A. B.; SOARES, S. M.; SANTOS, J. F.; SILVA, L. B. Ponto de corte para o WHOQOL-bref como preditor de qualidade de vida de idosos. Saúde Pública, v.48, n.3, p.390$397,2014$.

SOUZA, M.; MARCON, S. S.; BUENO, S. M. V.; CARREIRA, L.; BALDISSERA, V. D. A.. A vivência da sexualidade por idosas viúvas e suas percepções quanto à opinião dos familiares a respeito. Saúde e Sociedade, São Paulo, v.24, n.3, p.936944, 2015.

VAGETTI, G. C.; FILHO, V. C. B.; MOREIRA, N. B.; OLIVEIRA, V.; MAZZARDO, O.; CAMPOS, W.. Condições de saúde e variáveis sociodemográficas associadas à qualidade de vida em idosas de um programa de atividade física de Curitiba, Paraná, Sul do Brasil. Caderno de Saúde Pública, Rio de Janeiro, v.29, n.5, p.955-969, 2013.

VIEIRA, K. F. L.; REIS, I. D.; SEGUNDO, J. B. M.; FERNANDES, M. E.; MACDONALD, T. T. V.. Representações Sociais da Qualidade de Vida na Velhice. Psicologia: ciência e profissão, v.32, n.3, p.540-551, 2012.

VISENTIN, A.; MANTOVANI, M. F.; CAVEIÃO, C.; MENDES, T. A.; NEVES, A. S.; HEY, A. P.. Qualidade de vida de idosas hipertensas de uma instituição de longa permanência. Rene, v.16, n.2, p.218-225, 2015.

VITORINO, L. M.; PASKULIN, L. M. G.; VIANNA, L. A. C. Qualidade de vida de idosos da comunidade e de instituições de longa permanência: estudo comparativo. Revista LatinoAmericana de Enfermagem, v.21, 2013.

A CBPC - Companhia Brasileira de Produção Científica (CNPJ: 11.221.422/0001-03) detém os direitos materiais desta publicação. Os direitos referem-se à publicação do trabalho em qualquer parte do mundo, incluindo os direitos às renovações, expansões e disseminações da contribuição, bem como outros direitos subsidiários. Todos os trabalhos publicados eletronicamente poderão posteriormente ser publicados em coletâneas impressas sob coordenação da Sustenere Publishing, da Companhia Brasileira de Produção Científica e seus parceiros autorizados. Os (as) autores (as) preservam os direitos autorais, mas não têm permissão para a publicação da contribuição em outro meio, impresso ou digital, em português ou em tradução. 